\title{
Adaptive Aft Signature Shaping of a Low-Boom Supersonic Aircraft Using Off-Body Pressures
}

\author{
Irian Ordaz* and $\mathrm{Wu} \mathrm{Li}^{\dagger}$ \\ NASA Langley Research Center, Hampton, VA 23681
}

\begin{abstract}
The design and optimization of a low-boom supersonic aircraft using the state-of-theart off-body aerodynamics and sonic boom analysis has long been a challenging problem. The focus of this paper is to demonstrate an effective geometry parameterization scheme and a numerical optimization approach for the aft shaping of a low-boom supersonic aircraft using off-body pressure calculations. A gradient-based numerical optimization algorithm that models the objective and constraints as response surface equations is used to drive the aft ground signature toward a ramp shape. The design objective is the minimization of the variation between the ground signature and the target signature subject to several geometric and signature constraints. The target signature is computed by using a least-squares regression of the aft portion of the ground signature. The parameterization and the deformation of the geometry is performed with a NASA inhouse shaping tool. The optimization algorithm uses the shaping tool to drive the geometric deformation of a horizontal tail with a parameterization scheme that consists of seven camber design variables and an additional design variable that describes the spanwise location of the midspan section. The demonstration cases show that numerical optimization using the state-of-the-art off-body aerodynamic calculations is not only feasible and repeatable but also allows the exploration of complex design spaces for which a knowledge-based design method becomes less effective.
\end{abstract}

\section{Nomenclature}

Acronyms

$\begin{array}{ll}\text { BOSS } & =\text { boom optimization using smoothest shape (a computer code for low-boom design) } \\ \text { CFD } & =\text { computational fluid dynamics } \\ \text { DFO } & =\text { derivative-free optimization } \\ \text { DOE } & =\text { design of experiments } \\ \text { GBO } & =\text { gradient-based optimization } \\ \text { PLdB } & =\text { perceived loudness in decibels } \\ \text { psf } & =\text { pounds per square foot }\end{array}$

Symbols

$C_{L} \quad=$ coefficient of lift

$d p / p \quad=$ near-field pressure waveform

$X_{e} \quad=$ equivalent length

* Research Engineer, Aeronautics Systems Analysis Branch, Systems Analysis and Concepts Directorate.

${ }^{\dagger}$ Senior Research Engineer, Aeronautics Systems Analysis Branch, Systems Analysis and Concepts Directorate. 


\section{Introduction}

7 HE design and optimization of a low-boom supersonic aircraft using the state-of-the-art off-body 1 aerodynamics and sonic boom analysis has been a challenging problem as a result of the difficulty in the integration and automation of the surface and volume meshing that is required by computational fluid dynamics (CFD) and calculation of accurate off-body $d p / p$ measurements several body lengths below the aircraft. The off-body $d p / p$ that accounts for all three-dimensional aerodynamic effects of the aircraft is necessary for high-fidelity sonic boom analysis. Despite recent advances in the integration and automation of CFD off-body analysis [1], which makes the use of numerical optimization to shape the aft signature of a configuration feasible, the requirement for accurate off-body $d p / p$ measurements makes the design and optimization of low-boom aircraft computationally expensive.

The diagram in Fig. 1 shows several analysis and optimization approaches that have been published for the design of low-boom supersonic aircraft. Several publications [1-3] have used on-body aerodynamic analysis to perform derivative-free optimization (DFO) of sonic boom metrics, such as equivalent area, perceived loudness (PLdB) level, and signature features (i.e., initial, minimum, and maximum overpressure rise). Although this approach is less accurate, it is still useful because of the low computational expense that is associated with on-body aerodynamic CFD calculations.

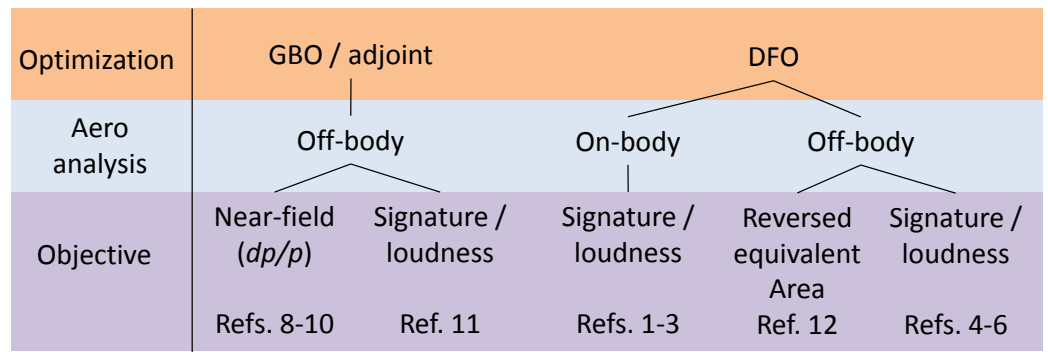

Figure 1. Current analysis and optimization approaches.

Makino and Kroo [4] used low-fidelity off-body aerodynamic calculations to conduct a robust optimization of a wing-body-tail configuration using an A-weighted decibel metric with a penalty for short pressure peak intervals, along with shape constraints for the ground signature. Off-body aerodynamic calculations can be more costly with CFD analysis but are also more accurate because they are able to fully capture the three-dimensional effects of the aircraft before propagation to the ground. Choi, Alonso, and Kroo [5] used a multi-fidelity approach to correct the low-fidelity linearized panel aerodynamics code with high-fidelity CFD solutions at the near-field locations to improve the computational efficiency for numerical optimization. Other researchers have opted to exclusively use the more costly high-fidelity off-body CFD calculations for simple configurations, such as wing-body configurations [6].

The adjoint methodology (see the review in [7]) has been a game changer because it allows the efficient and accurate calculation of the gradient of the objective for a large number of design variables. The gradient information makes the use of gradient-based optimization (GBO) method feasible for CFDbased shape optimization with a large number of design variables. The GBO method, with the use of adjoint-based gradient information, has been applied to the numerical optimization of a low-boom supersonic aircraft [8-10] with near-field $d p / p$ target matching as the objective. Adjoint design with ground signature matching as the optimization objective has also been demonstrated by Rallabhandi [11] for a complete aircraft. Although adjoint-based optimization has great potential for the development of low-boom supersonic aircraft, the technology needs to be further tested for practical applications. A recent study by Aftosmis, Nemec, and Cliff [8] demonstrates that an adjoint off-body design optimization method is capable of generating a low-boom configuration with a fully-shaped ground signature. In this study, the optimization objective is to match an off-body $d p / p$ target.

Typically, for inverse design optimizations, the objective is the minimization of the total error between the desired target and the equivalent area, near-field $d p / p$, or signature of a design. In general, an inverse design optimization problem may not have a solution because the target may not be realizable. This is one of the main challenges that is associated with inverse low-boom design optimization. Improved target-generation techniques for equivalent area distributions and signatures with desired lowboom characteristics have made the inverse design and optimization of a low-boom aircraft possible. Li and Rallabhandi [12] demonstrated the inverse design of a low-boom supersonic concept that matches a reversed equivalent area target. The reversed equivalent area is a conversion of the CFD $d p / p$ at several body lengths below the aircraft and captures the three-dimensional effects of the aircraft. Unlike the classical equivalent area that is generated by Mach angle cuts based on surface pressure, the reversed 
equivalent area has the same ground signature as the aircraft when a high-fidelity off-body sonic boom analysis is used to compute the ground signature. As a result, by comparing the reversed equivalent area distributions with a desired target, a designer can identify the regions of the vehicle where shape optimization can lead to an improved low-boom design. Matching a reversed equivalent area target by volume shaping with BOSS [13] is effective and has been demonstrated to produce a good match up to approximately 90 percent of the vehicle equivalent length [12]. This inverse design method reduces a high-fidelity low-boom design problem to aft shaping of the last 10 percent of the vehicle equivalent length.

For this work, the reversed equivalent area matching approach was used to shape the front 90 percent of the vehicle equivalent length; then a novel numerical optimization method was applied to shape the aft signature. The mismatch between the reversed equivalent area and a low-boom equivalent area target is used to identify the appropriate design region on the aircraft for aft shaping and to determine the parameterization scheme for low-boom shape optimization. The parameterization scheme aims at tailoring the aft lift distribution of the vehicle to obtain a fully shaped ground signature. The improvement of the aft signature is achieved by minimizing the difference between the aft signature and a linear target. Two examples are included to illustrate the repeatability of the overall aft-shaping optimization approach. One of the optimized configurations is reanalyzed with several CFD meshes of various resolutions to show that the ground signature of the optimized configuration is insensitive to mesh resolution.

The automated high-fidelity sonic boom analysis process is reviewed in section II. The problem definition, including baseline definition, parameterization scheme, and optimization objectives and constraints are described in section III. Optimization results are given in section IV, and concluding remarks are provided in section $\mathrm{V}$.

\section{Integrated Off-Body Sonic Boom Analysis}

This section reviews the analysis tools and modeling techniques that are used in this paper, including synthesis of analysis tools, aerodynamic analysis, and sonic boom propagation. The geometry generation, parameterization, and deformation tools, as well as the CFD and sonic boom analysis codes, are integrated in ModelCenter [14] for automated off-body sonic boom analysis. The integrated analysis process illustrated by the flowchart in Fig. 2 is documented in Ref. 1. The built-in optimization tools in ModelCenter (e.g., Design Explorer) allow a user to easily set up optimizations with the objective and constraints generated from the integrated analysis codes in ModelCenter. For easy reference, the following two subsections review the key analysis tools and methods that are used for automated high-fidelity sonic boom analysis.

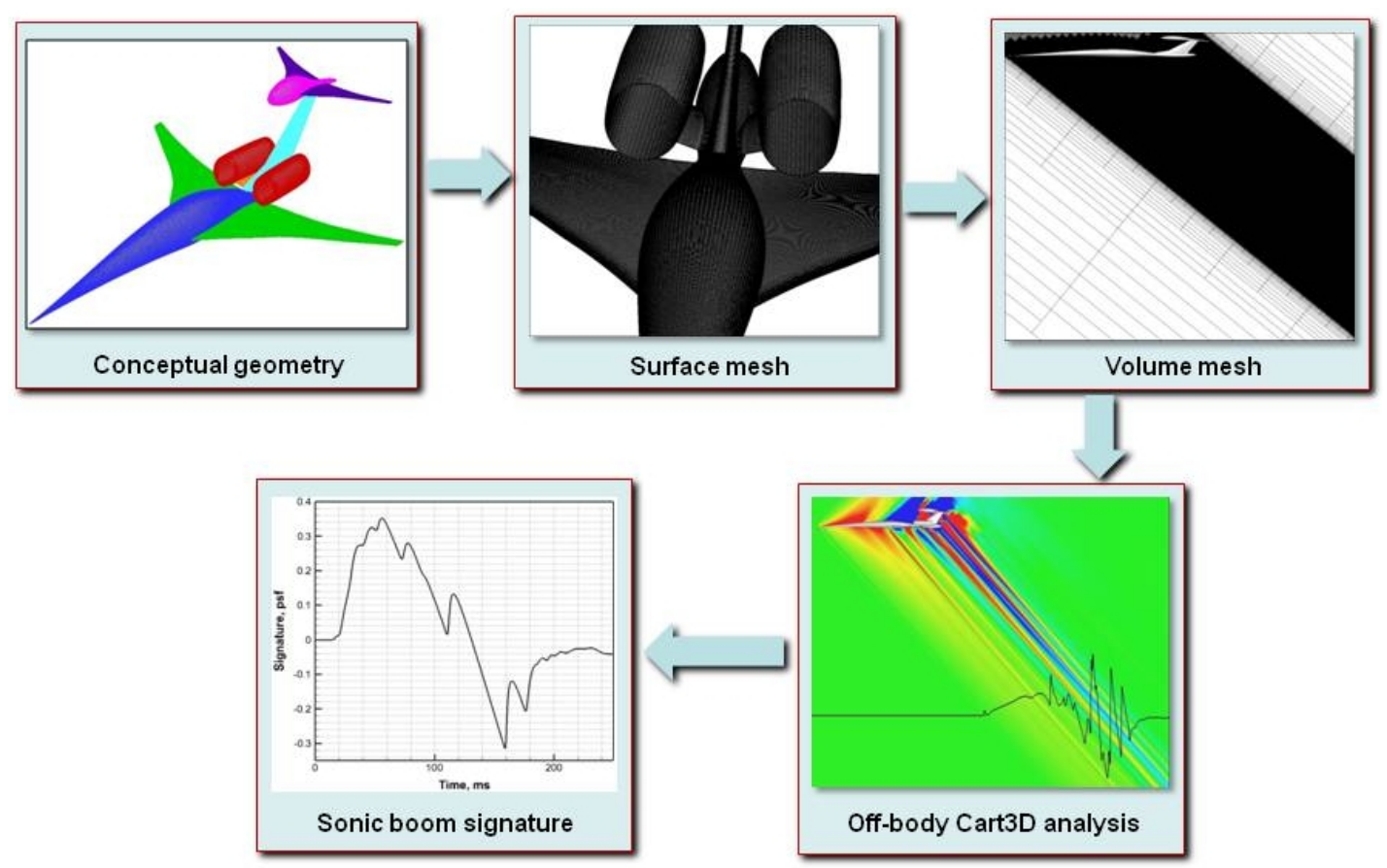

Figure 2. Integrated off-body sonic boom analysis process. 


\section{A. CFD Analysis for Off-Body Pressures}

All CFD analysis results in this paper are generated with Cart3D, which is an inviscid CFD analysis package that is geared toward conceptual and preliminary aerodynamics design $[15,16]$. The package allows for quick and automated Cartesian-based volume mesh generation and CFD solutions for complex geometries. It includes numerous tools that facilitate the conversion and import of various geometry formats. In addition, it is highly scalable and can be run on multiprocessor systems. For postprocessing, Cart3D can export plane cuts for flow parameters at arbitrary locations, as well as line cuts for pressures at off-body locations.

Figure 3 shows a sample pressure contour on the symmetry plane that was obtained with Cart3D for a low-boom concept with flow-through nacelles at Mach 1.6 with an angle of attack of 0.49 deg. The volume mesh is rotated by Mach angle to align the shocks with the computational grid and consists of stacked refinement boxes to improve mesh efficiency. The automated process of meshing and computing the off-body $d p / p$ for a conceptual geometry was implemented in ModelCenter. The automated Cart3D off-body analysis process, with a stretched and rotated grid, was verified with USM3D [17] by using a sheared and stretched grid [18]. A comparison of the Cart3D and USM3D $d p / p$ solutions shows a good agreement at three body lengths below the aircraft (see Fig. 4). The shock structure that is calculated with Cart3D agrees with the USM3D solution, and the differences in the magnitudes of the peaks are most likely the result of the different meshing and refinement strategies that were used with the two codes.

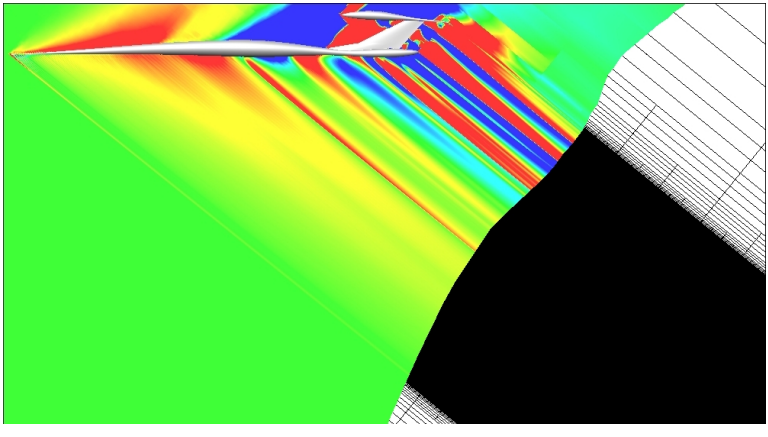

Figure 3. Cart3D symmetry plane pressure solution with stacked and rotated volume mesh.

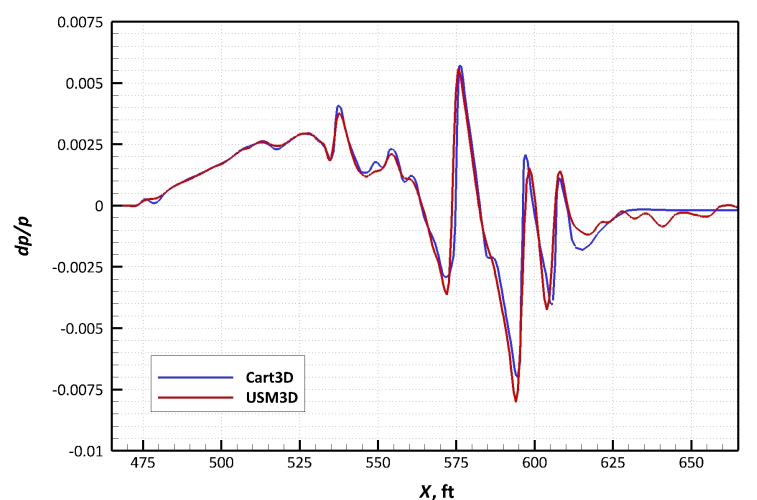

Figure 4. Verification of automated Cart3D offbody analysis process by USM3D.

\section{B. Sonic Boom Propagation}

The sonic boom analysis is conducted by using the Cart3D off-body pressure at a constant $z$-location. This off-body waveform is then propagated by using a sonic boom propagation code called sBOOM [19] to obtain the ground signatures that are shown in Fig. 5. By solving the augmented Burgers equation, sBOOM takes into account nonlinearity, molecular relaxation, and thermo-viscous absorption and is capable of generating off-track signatures for off-track $d p / p$ distributions. A comparison of the ground signatures that are generated by $\mathrm{SBOOM}$ and $\mathrm{PCBOOM}$ is provided in Fig. 5; this comparison demonstrates good agreement between the two codes when absorption and molecular relaxation are not used by sBOOM. The figure also shows that absorption and molecular relaxation have significant effects on the ground signature. The finite rise time of the ground signature when absorption and molecular relaxation are used by sBOOM leads to more accurate PLdB calculations. The sonic boom propagation is performed by using a reflection value of 1.9 . 


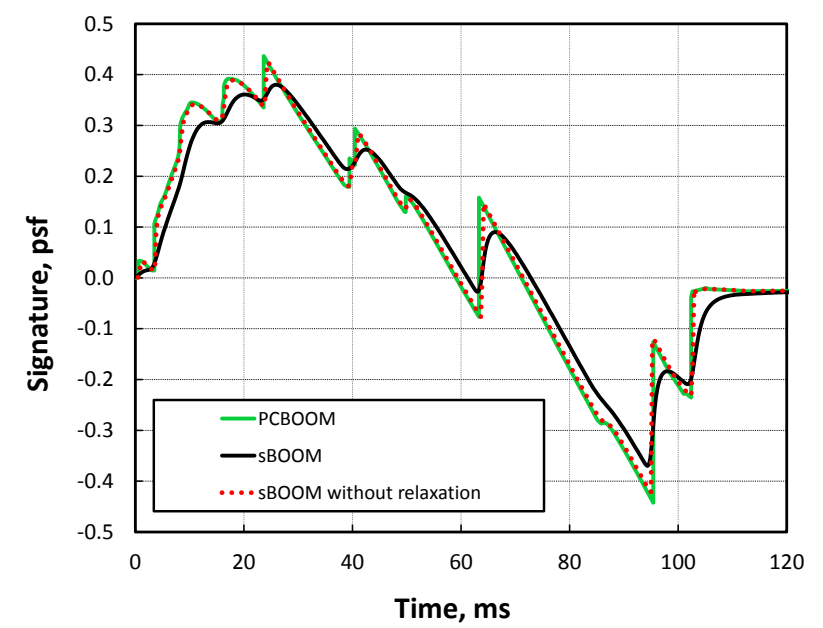

Figure 5. Ground signature comparison of sBOOM and PCBOOM.

\section{Formulation of the Optimization Problem}

The high computational cost of off-body pressure calculations using CFD makes automated numerical optimization a challenging task. A good understanding of both the design space and the key metrics is needed to properly define a parameterization scheme that can be used to effectively improve the design. The effectiveness of a parameterization scheme can be quantified by the ratio of the improvement in the objective function to the total number of analysis function calls (in this case, CFD runs). Furthermore, the automated Cart3D analysis potentially may fail because of the difficulty in generating a volume mesh for a conceptual geometry (defined by grid points for each of the aircraft components). However, a failed CFD run could have a detrimental effect on most numerical optimization codes. As a result, the high-fidelity low-boom optimization problem requires an optimization code that is insensitive to failed function evaluations. The optimization code Design Explorer (which is available within ModelCenter) uses surrogate models to approximate the objective and the constraints. It repeatedly solves the optimization problem with the surrogate models, while continuously updating the surrogate models with new function evaluations. This optimization code is known for its capability to solve complex physics-based engineering design problems with a high tolerance for failed function evaluations and is used to solve the high-fidelity low-boom optimization problems in this work. Nonetheless, care must be taken so that the number of failed cases is not large enough to adversely affect the accuracy of the surrogate models for the objective and the constraints.

Two optimization cases with different baseline configurations and geometric constraints are presented to verify the repeatability of the parametric scheme and the optimization approach. The first case documents the parameterization scheme and the rationale for the decisions that were made. The second case uses a different baseline and geometric constraints to verify that the overall aft-shaping optimization approach is repeatable and that multiple design paths exist for the development of low-boom configurations with fully shaped ground signatures. The general configuration for both optimization baselines is shown in Fig. 6.

These two baselines were derived from the same low-boom configuration that was introduced in Ref. [12]. The original low-boom configuration was designed for a cruise Mach number of 1.6, a cruise altitude of $45,000 \mathrm{ft}$, and a fixed angle of attack of $0.5 \mathrm{deg}$. The resulting cruise weight of 27,000 lb was lower than intended. The ground signature for the same configuration at an angle of attack of 0.8 (which corresponds to a cruise weight of approximately 33,000 lb) exhibits some undesirable features in the aft portion. A series of optimization runs with various optimization formulations was conducted without much success, to improve the aft signature for the configuration at an angle of attack of 0.8. However, one of the solutions did have an aft signature shape that was easier to reshape to recover the desirable low-boom features. Both the original configuration and this semi-optimized configuration were used as the starting points for the inverse design process with reversed equivalent area matching (as documented in Ref. [12]). The two baseline configurations were the final results of the reversed equivalent area matching. The main wings, vertical tails, pylons, and nacelles are identical in both baselines, while the fuselages, tail pods, and horizontal tails are different. The differences in the baselines for these components are given in the appendix (Figs. A-1 to A-3). These figures have been scaled in the $z$-coordinate for easier visualization. 
The parameterization scheme that was used in both optimization cases is shown in Fig. 7 . The scheme consists of eight total design variables on the horizontal tail. Seven of these design variables (var1 through var7) control the camber at three airfoil locations. Three camber control points were placed at the leading edge, mid chord, and trailing edge of the root and midspan airfoil locations. Another camber control point was placed at the trailing edge of the tip airfoil. Lastly, the spanwise location (var8) of the midspan airfoil section was allowed to vary. A rapid shape parameterization tool called Proteus [20] was used to implement this parametric scheme.

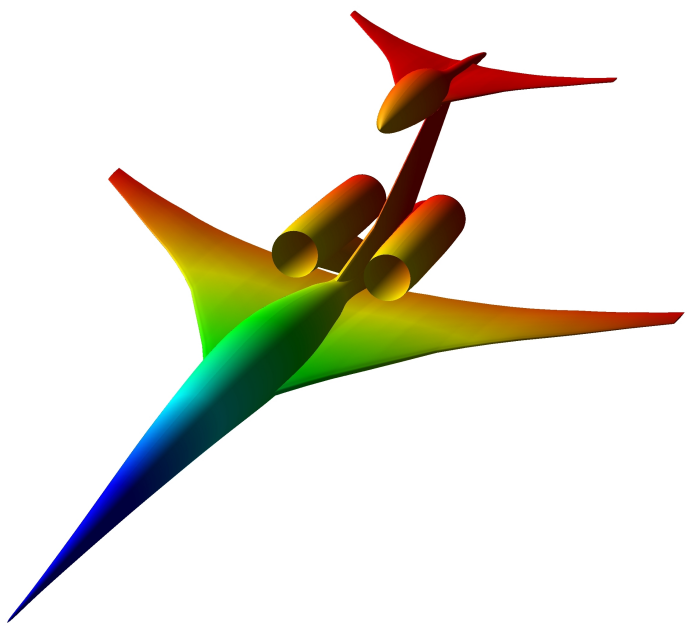

Figure 6. Baseline configuration.

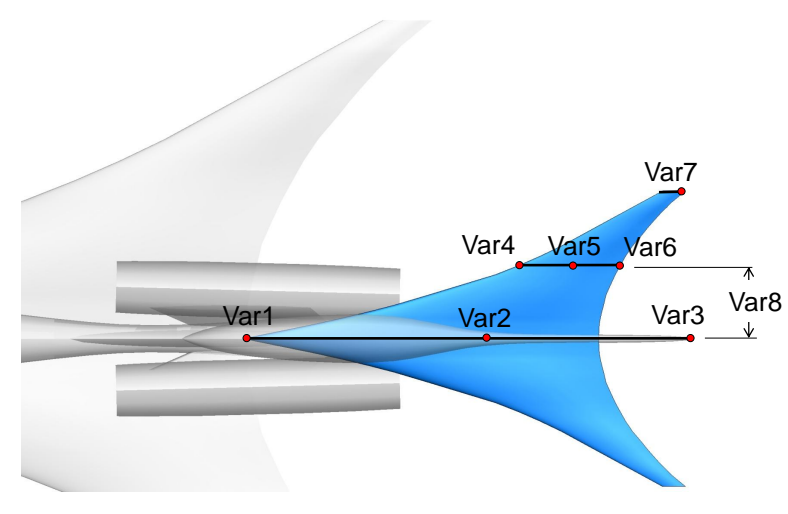

Figure 7. Parametric scheme of horizontal tail.

The initial design variables that correspond to baselines $\# 1$ and $\# 2$, as well as the design variable ranges, are given in the appendix in tables A-1 and A-2, respectively. These variable ranges were customarily chosen to ensure that the entire design space resulted in realistic tail shapes. The optimization objective was to minimize the total variation between the aft ground signature and a linear target signature. The linear target was obtained by computing the least-squares fitting of the aft portion of the ground signature, which ranged from 80 to $130 \mathrm{~ms}$. Two constraints were used to bound the range of the ground signature by that of the baseline. That is, the range for the ground signature was from -0.2086 to $0.3991 \mathrm{psf}$ and from -0.2188 to $0.3724 \mathrm{psf}$ for the first and second optimization cases, respectively. Lift, drag, and PLdB were tracked but not constrained to allow greater design flexibility. Although the configuration lift potentially can decrease during optimization iterations, the angle of attack was chosen to be greater than actually required. As a result, the final lift for the design had to be checked to ensure that it met the original requirement of a start cruise weight of at least 33,000 lb.

\section{Results}

The first and second optimization problems were solved with Design Explorer in ModelCenter with a total of 180 and 213 high-fidelity Cart3D off-body analyses (including several failed CFD runs), respectively. An off-body $d p / p$ analysis at three body lengths below the aircraft was calculated for each optimization analysis case. The Cart3D analysis was conducted by using 64 cores on a computer system that consisted of eight nodes with two quad-core processors (Intel Xeon E5462@ $2.80 \mathrm{GHz}$ ) per node. The computational mesh that was used for the CFD analysis for the first and second optimization case consisted of approximately 20 million and 25 million cells, respectively. Each Cart3D analysis that was used to obtain off-body $d p / p$ measurements at three body lengths below the aircraft for optimization cases \#1 and \#2 required approximately $1 \mathrm{hr}$ and $38 \mathrm{~min}$, and $1 \mathrm{hr}$ and $49 \mathrm{~min}$, respectively, to complete. This demonstrates a turnaround time for the optimization cases that ranged between 12 and 15 days (considering the failed cases), or approximately two weeks.

The convergence histories for the objective residual are shown in the appendix in Figs. A-4 and A-5. Note that Design Explorer used 64 function evaluations (involving 64 CFD analyses) to construct the initial surrogate model for the objective and constraint functions. The baseline, design of experiments (DOE) data, and optimization solutions for the two optimization problems are plotted in Figs. 8 and 9. These figures, which show scatter plots of the lift-to-drag ratio as a function of PLdB, are used to identify promising low-boom candidate configurations. The optimum solution was chosen based on engineering 
judgment by taking into account the shape of the signature, the lift coefficient, the lift-to-drag ratio, and the PLdB for the generated configurations. In general, the optimum solution has a PLdB value that is close to the lowest PLdB level observed and a $C_{L}$ value that is close to the baseline, along with a shaped aft signature. Among the configurations with similar aft signature shapes, the configuration with the highest $C_{L}$ is selected as the optimum solution, which is labeled "Optimum" in Figs. 8 and 9. For comparison, the DOE configuration with the lowest PLdB value was selected, along with another configuration that had a better lift-to-drag ratio and a PLdB value that was close to the lowest value. These two configurations are labeled in Figs. 8 and 9. The best DOE solution is included for comparison to determine whether the optimization formulation is effective in improving the aft signature.

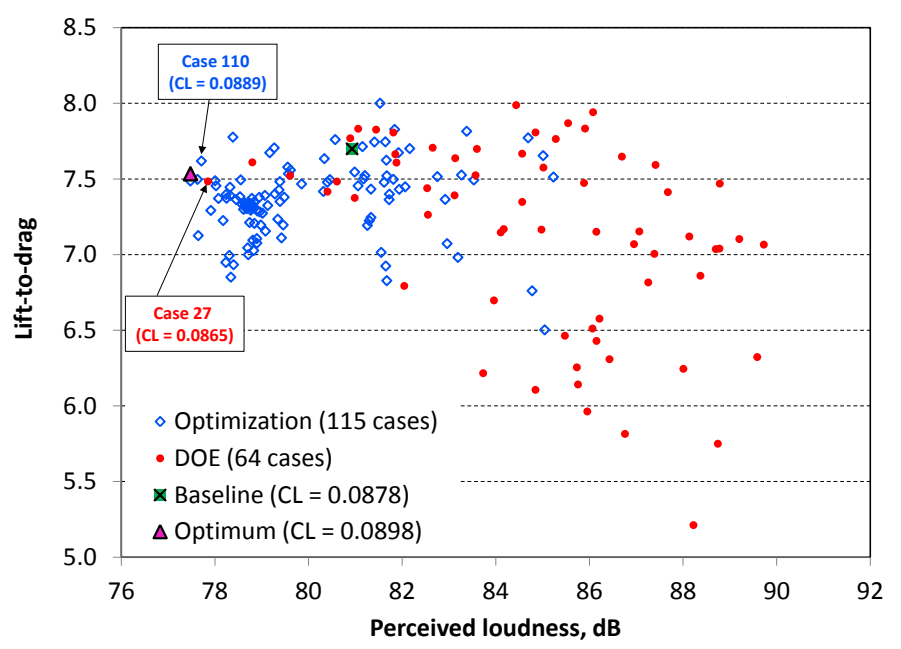

Figure 8. Comparison of perceived loudness and lift-to-drag ratio for optimization \#1.

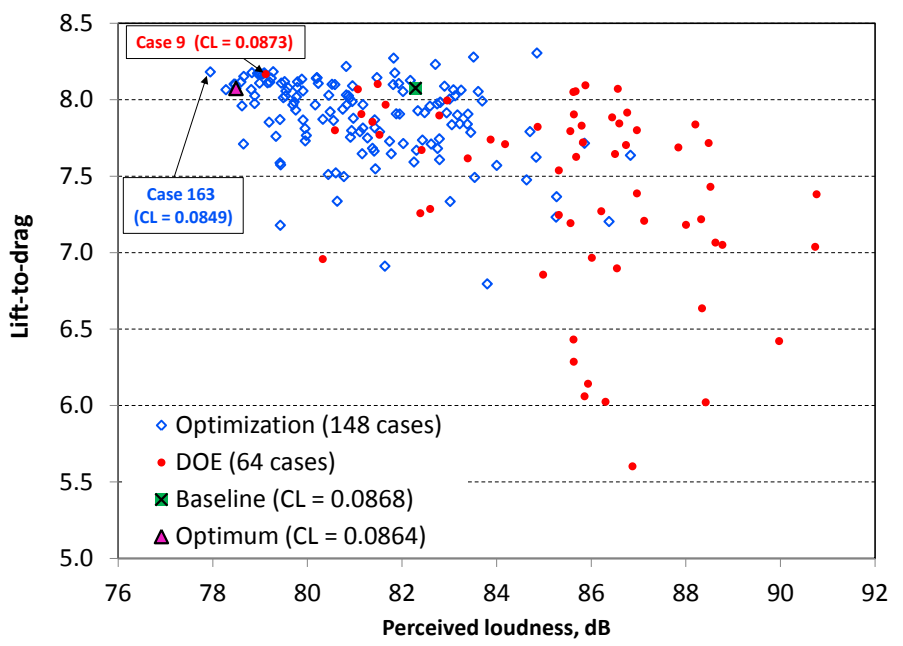

Figure 9. Comparison of perceived loudness and lift-to-drag ratio for optimization \#2.

The signatures, along with the coefficients of lift and the PLdB for the three selected configurations, are shown in Figs. 10 and 11 for the optimizations of the two baselines, respectively. For the optimization of baseline \#1, case 143 was chosen because of its desirable signature with a flat shock structure in the aft region of the aircraft. In addition, it provided the highest coefficient of lift and the lowest PLdB levels with minimal compromise in the lift-to-drag ratio. In this case, the optimization formulation and the parametric scheme accomplished the intended goals of aft signature shaping: the optimum configuration \#2 can fly with approximately 4 percent higher cruise weight for a better aft signature shape with a lower PLdB level than the best DOE solution. For the optimization of baseline \#2, case 213 was chosen as the optimum configuration because of its desirable aft signature shape. Despite the similarly desirable signature shape of DOE case 9, this configuration was not chosen because of its higher PLdB level. However, this decision is subjective because case \#9 was analyzed at a cruise weight that was 1 percent higher than that for case 213. Both configurations (\#9 and \#213) have equally well-shaped aft signatures. Note that low-boom shaping is not a deterministic process and that both optimization cases 
provided several low-boom candidates. Furthermore, the second optimization and the design case that are presented in Ref. [12] show that with a good parameterization scheme a well-shaped aft signature may be achievable with a DOE method for tail lift tailoring.

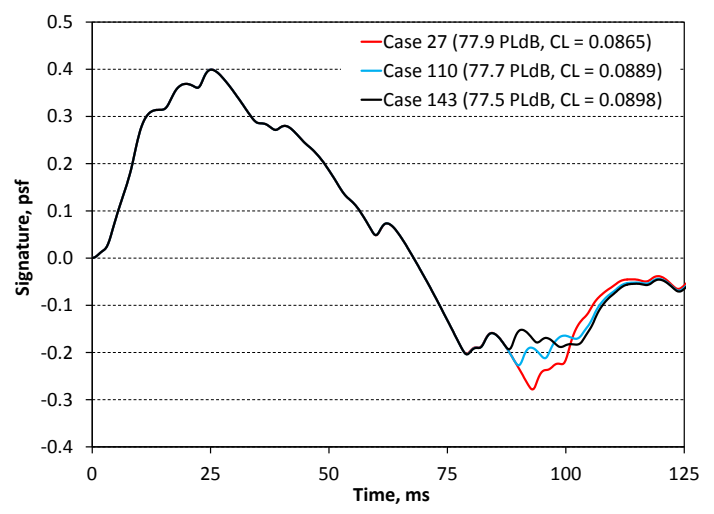

Figure 10. Comparison of promising candidates from optimization \#1.

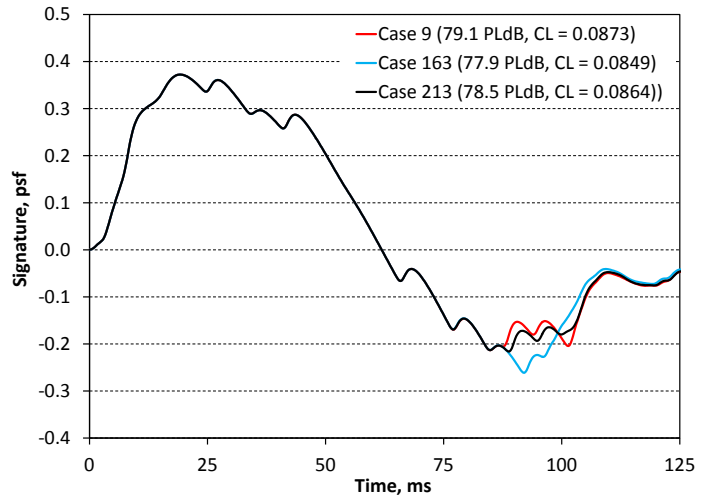

Figure 11. Comparison of promising candidates from optimization \#2.

Figures 12 to 17 provide a comparison of the analysis results for the pre-BOSS, baseline, and optimum configurations. These will help to better understand the process of achieving a well-shaped aft signature for a low-boom configuration. The first pre-BOSS configuration was the result of a trial optimization to improve the low-boom configuration that is documented in Ref. [3] at an angle of attack of 0.8 deg, while the second pre-BOSS configuration was the original low-boom configuration that is presented in Ref. [3]. These two configurations serve as the starting points for a systematic aft signature shaping process, which is the inverse design of matching a reversed equivalent area up to 85 percent (approximately $118 \mathrm{ft}$ ) of the vehicle equivalent length of $140 \mathrm{ft}$, as well as the numerical optimization to minimize the difference of the aft signature and an adaptive linear target.

A comparison of the reversed equivalent areas that were calculated from $d p / p$ measurements at three body lengths below the aircraft is shown in Figs. 12 and 13 for the two optimization cases. The inverse design process matches the reversed equivalent area to a low-boom target up to $118 \mathrm{ft}$ of the vehicle equivalent length. A close inspection of Figs. 12 and 13 reveals that the targets for the inverse design are different in these two cases, with a target equivalent area of $46 \mathrm{ft}^{2}$ in Fig. 12 versus that of $44.8 \mathrm{ft}^{2}$ in Fig. 13 at $X_{e}=118 \mathrm{ft}$. Also, the difference between the aft reversed equivalent areas of the baseline and the optimum configuration for optimization case \#1 is greater than that for optimization case \#2. Figures 14 and 15 compare the $d p / p$ at three body lengths below the configuration; these are used to calculate the reversed equivalent areas. The $d p / p$ for the optimized configurations have a shock structure that is similar to that of the baselines, but the shock strengths have been optimized to achieve some degree of shock cancellation.

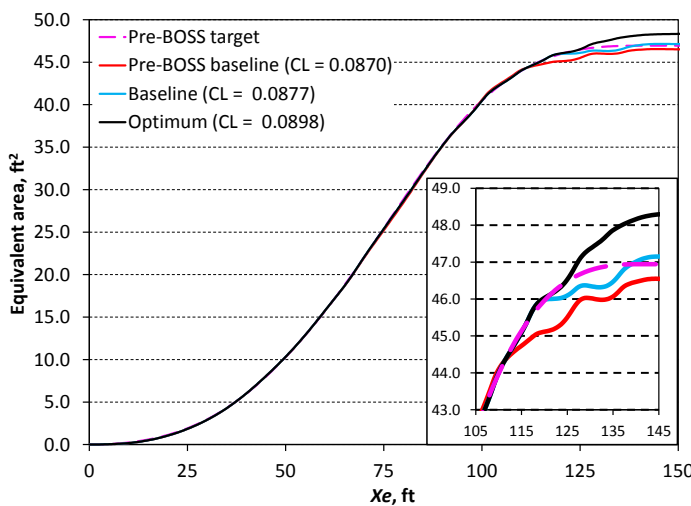

Figure 12. Comparison of reversed equivalent areas for optimization \#1.

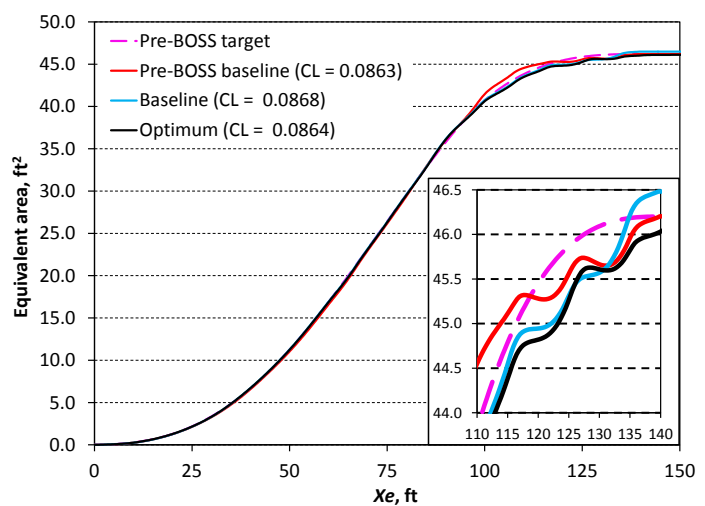

Figure 13. Comparison of reversed equivalent areas for optimization \#2. 


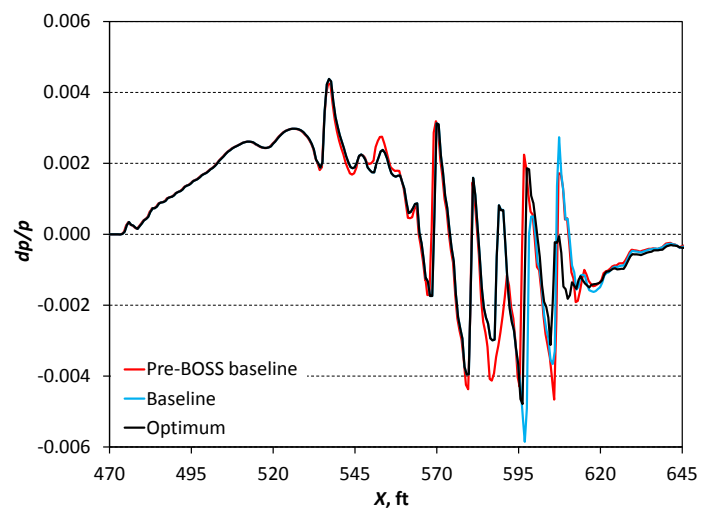

Figure 14. Comparison of $d p / p$ for optimization \#1.

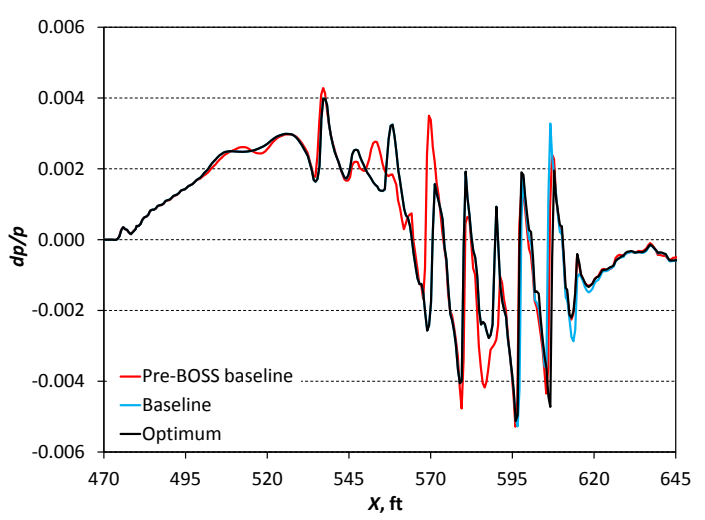

Figure 15. Comparison of $d p / p$ for optimization \#2.

A comparison of the ground signatures of the pre-BOSS, baseline, and optimum configurations is provided in Figs. 16 and 17. Recall that the optimization objective was to minimize the pressure variation at the aft portion of the signature, from 80 to $130 \mathrm{~ms}$. Note that a novel feature of the numerical optimization formulation is the change of the linear target between the optimization cases. Because the linear target is the least-squared fit of the aft signature by a linear function, different aft signatures lead to different targets. The implicit optimization objective is to make the aft signature as flat as possible over the design range. The plots indicate that the two-shock structure in the baseline, which is denoted by the blue curve, has been broken into several smaller shocks. In the first optimization case, the minimum overpressure was reduced by nearly one-third in magnitude.

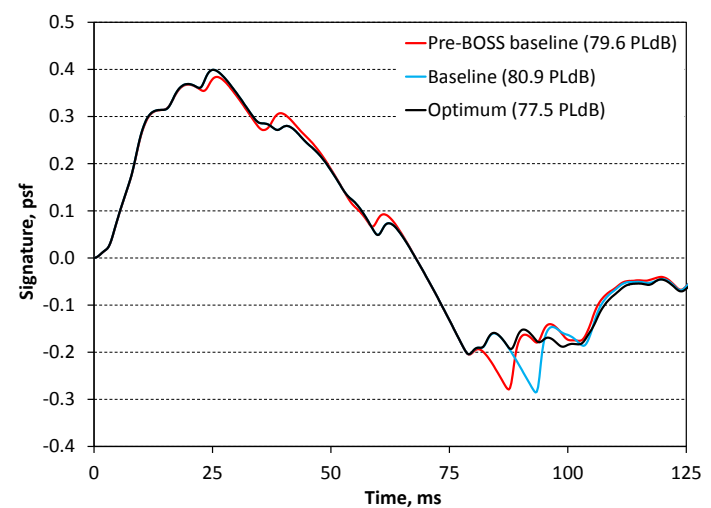

Figure 16. Comparison of signatures for optimization \#1.

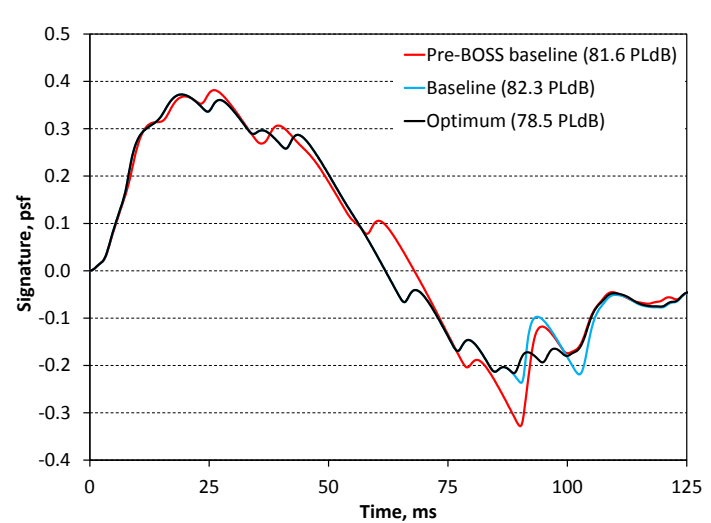

Figure 17. Comparison of signatures for optimization \#2.

The design parameter settings for the optimized configurations are given in the appendix in tables A-1 and A-2. The baseline and optimum tail geometries are compared in Fig. A-6. These figures have been scaled in the $z$-coordinate for easier visualization. Despite starting with similar horizontal tails for the two baselines (see Fig. A-2), the two optimum horizontal tails are quite different (see Fig. A-6). This further indicates that low-boom configurations are not unique, and the "abundance" of low-boom configurations also demonstrates the feasibility of adding additional design constraints/objectives to the low-boom optimization problem. The parametric scheme with eight design variables, along with the adaptive linear target for the aft signature, proves to be highly effective in achieving a well-shaped aft signature for a configuration with a T-tail.

One important issue in CFD-based optimization is whether the numerical optimizer is exploiting weaknesses of the numerical algorithm coupled with the CFD solver to produce analysis results that do not correctly capture the flow physics (or the Euler flow in this case). An example of this is when the optimizer moves shocks to regions where the volume mesh is coarse. Extensive studies have been conducted to ensure that the automated analysis result is as accurate as the Cart3D solution with a mesh of the highest cell density that can be obtained with the available computing resources. A study has also been performed to ensure that the automated Cart3D $d p / p$ solution agrees with a solution that 
was obtained from USM3D (see Fig. 4). Verifying that the obtained $d p / p$ measurements at three body lengths below a configuration via the automated Cart3D analysis process is the true Euler solution for the configuration can be difficult. The parameter-insensitivity of the obtained solution is the next best metric for verifying whether the obtained analysis results are credible. Here consistency means that the obtained numerical solution is insensitive to algorithmic parameter changes (e.g., the CFD mesh density). This method of verification does not prove that the obtained numerical solution is physically accurate, but it improves the credibility of the analysis results. A sensitivity analysis of the optimum configuration \#2 with respect to mesh density is provided in the appendix in Figs. A-7 and A-8. The curve that is labeled "Analysis" demonstrates the results for the same volume mesh density (approximately 25 million cells) as the automated Cart3D analysis process for the two optimization cases, which results in a pressure distribution of approximately 418 points at three body lengths below the aircraft. The volume mesh size was increased from 25 million cells to 47 and 91 million cells for the mesh sensitivity study, which resulted in an increase in the number of data points for the pressure distributions to 527 and 695, respectively. The shocks in the $d p / p$ and the ground signature shapes are essentially the same, except for minor differences (see Figs. A-7 and A-8 in the appendix). The sensitivity study provides some credibility that the ground signature of the optimum configuration is fully shaped under the inviscid fluid dynamics analysis.

\section{Concluding Remarks}

An optimization approach has been presented for the design of a low-boom supersonic concept. The work demonstrated that optimization for low-boom characteristics may have multiple solutions. This work has demonstrated that the numerical optimization of a low-boom concept with high-fidelity offbody CFD solutions is feasible without an adjoint solver. Furthermore, the work has shown that a successful aft signature optimization can be performed with a simple yet effective parametric scheme that consists of only a few design variables (i.e., eight variables) and an adaptive linear target for the aft signature.

\section{References}

${ }^{1}$ Ordaz, I. and Rallabhandi, S. K., "Boom Minimization Framework for Supersonic Aircraft Using CFD Analysis," AIAA-2010-1506, January 2010.

${ }^{2}$ Rallabhandi, S. K., Li, W., and Geiselhart, K., "Boom-Constrained Drag Minimization for Design of Supersonic Concepts," AIAA-2010-844, January 2010.

${ }^{3} \mathrm{Li}$, W., Shields, E., and Geiselhart, K., "A Mixed-Fidelity Approach for Design of Low-Boom Supersonic Aircraft," AIAA-2010-845, January 2010.

${ }^{4}$ Makino, Y. and Kroo, I. M., "Robust Objective Functions for Sonic-Boom Minimization," Journal of Aircraft, Vol. 43, No. 5, 2006, pp. 1301-1306.

${ }^{5}$ Choi, S., Alonso, J. J., and Kroo, I. M., "Multifidelity Design Optimization of Low-Boom Supersonic Jets," Journal of Aircraft, Vol. 45, No. 1, 2008, pp. 106-118.

${ }^{6}$ Makino, Y., Watanuki, T., Kubota, H., Aoyama, T., and Iwamiya, T., "Low-Boom Design Method by Numerical Optimization," AIAA-98-2246, June 1998.

${ }^{7}$ Giles, M. B. and Pierce, N. A., "An Introduction to the Adjoint Approach to Design," Flow, Turbulence and Combustion, Vol. 65, No. 3-4, 2000, pp. 393-415. 2011.

${ }^{8}$ Aftosmis, M., Nemec, M., and Cliff, S., "Adjoint-Based Low-boom Design with Cart3D," AIAA-2011-3500, June

${ }^{9}$ Nemec, M. and Aftosmis, M., "Parallel Adjoint Framework for Aerodynamic Shape Optimization of ComponentBased Geometry," AIAA-2011-1249, January 2011.

${ }^{10}$ Nadarajah, S., Jameson, A., and Alonso, J., "An Adjoint Method for the Calculation of Remote Sensitivities in Supersonic Flow," AIAA-2002-0261, January 2002.

${ }^{11}$ Rallabhandi, S. K., "Sonic Boom Adjoint Methodology and its Applications," AIAA-2011-3497, June 2011.

${ }^{12} \mathrm{Li}, \mathrm{W}$. and Rallabhandi, S. K., "Inverse Design of Low-Boom Supersonic Concepts Using Reversed Equivalent Area Targets," AIAA-2011-3498, June 2011.

${ }^{13} \mathrm{Li}$, W., Shields, E., and Le, D., "Interactive Inverse Design Optimization of Fuselage Shape for Low-Boom Supersonic Concepts," AIAA-2008-136, January 2008.

14 "ModelCenter and Optimization Tools," Phoenix Integration, http://www.phoenix-int.com/ [cited October 2011].

${ }^{15}$ Aftosmis, M., "Cart3D Resource Website," http://people.nas.nasa.gov/ aftosmis/cart3d/cart3Dhome.html [cited October 2011].

${ }^{16}$ Aftosmis, M., Berger, M. J., and Adomavicius, G., "A Parallel Multilevel Method for Adaptively Refined Cartesian Grids with Embedded Boundaries," AIAA-2000-0808, January 2000.

17 "USM3D Users Manual, Version 6.0," http://tetruss.larc.nasa.gov/usm3d/ [cited October 2011].

${ }^{18}$ Campbell, R. L., Carter, M. B., and Deere, K. A., "Efficient Unstructured Grid Adaptation Methods for Sonic Boom Prediction," AIAA-2008-7327, August 2008.

${ }^{19}$ Rallabhandi, S. K., "Advanced Sonic Boom Prediction Using Augmented Burger's Equation," AIAA-2011-1278, January 2011.

${ }^{20} \mathrm{Li}$, W., "Rapid Parameterization Schemes for Aircraft Shape Optimization," AIAA No. Pending, January 2012. 


\section{Appendix: Additional Information}

This section contains additional information associated with the work presented. Included are the optimization variable constraints and optimized parameter values, baseline and optimized geometry comparisons, as well as a mesh sensitivity study.

Table A-1. Design Variables and Constraints for Optimization \#1

\begin{tabular}{lcccc}
\hline Variables & Baseline & Minimum & Maximum & Optimum \\
\hline \hline Camber1 (Var1) & 0 & -0.60 & 0.40 & 0.3726 \\
Camber2 (Var2) & 0 & -0.30 & 0.30 & 0.3 \\
Camber3 (Var3) & 0 & -0.40 & 0.20 & -0.1246 \\
Camber4 (Var4) & 0 & -0.20 & 0.20 & -0.0625 \\
Camber5 (Var5) & 0 & -0.20 & 0.20 & -0.1640 \\
Camber6 (Var6) & 0 & -0.15 & 0.15 & -0.1447 \\
Camber7 (Var7) & 0 & -0.10 & 0.10 & 0.0277 \\
Location (Var8) & 0.5 & 0.30 & 0.70 & 0.4875 \\
\hline
\end{tabular}

Table A-2. Design Variables and Constraints for Optimization \#2

\begin{tabular}{lcccc}
\hline Variables & Baseline & Minimum & Maximum & Optimum \\
\hline \hline Camber1 (Var1) & 0 & -0.10 & 0.60 & 0.6 \\
Camber2 (Var2) & 0 & -0.20 & 0.30 & 0.2873 \\
Camber3 (Var3) & 0 & -0.30 & 0.15 & 0.15 \\
Camber4 (Var4) & 0 & -0.20 & 0.20 & -0.0789 \\
Camber5 (Var5) & 0 & -0.15 & 0.15 & -0.1089 \\
Camber6 (Var6) & 0 & -0.15 & 0.15 & -0.0867 \\
Camber7 (Var7) & 0 & -0.20 & 0.20 & -0.0148 \\
Location (Var8) & 0.5 & 0.30 & 0.70 & 0.5406 \\
\hline
\end{tabular}

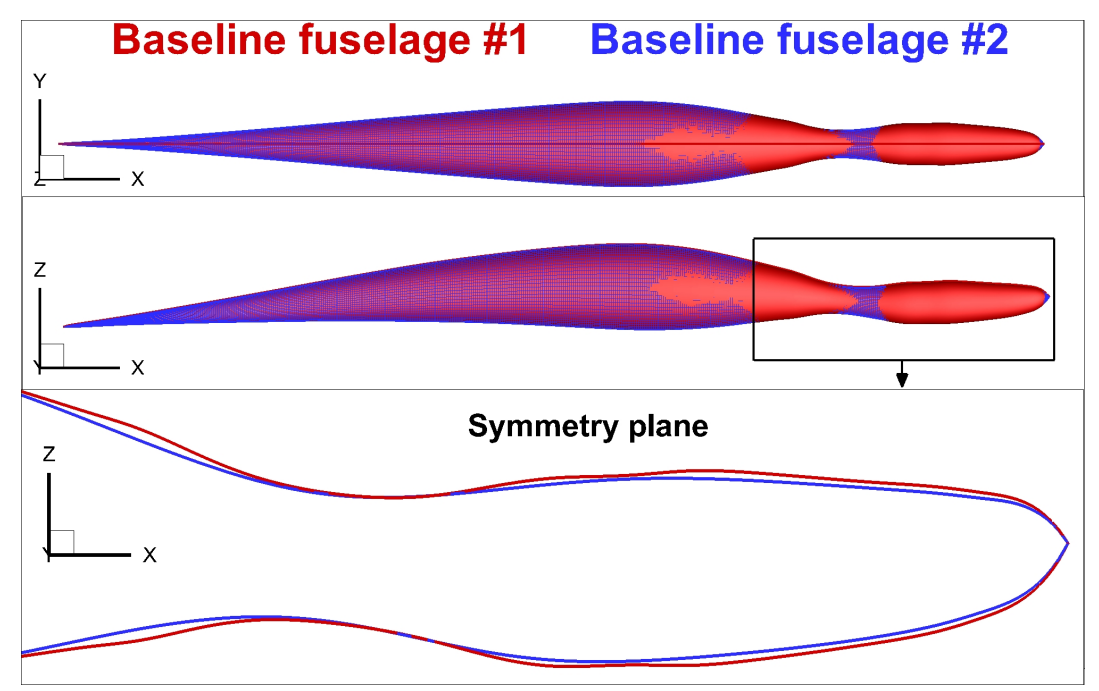

Figure A-1. Comparison of baseline fuselage configurations. 


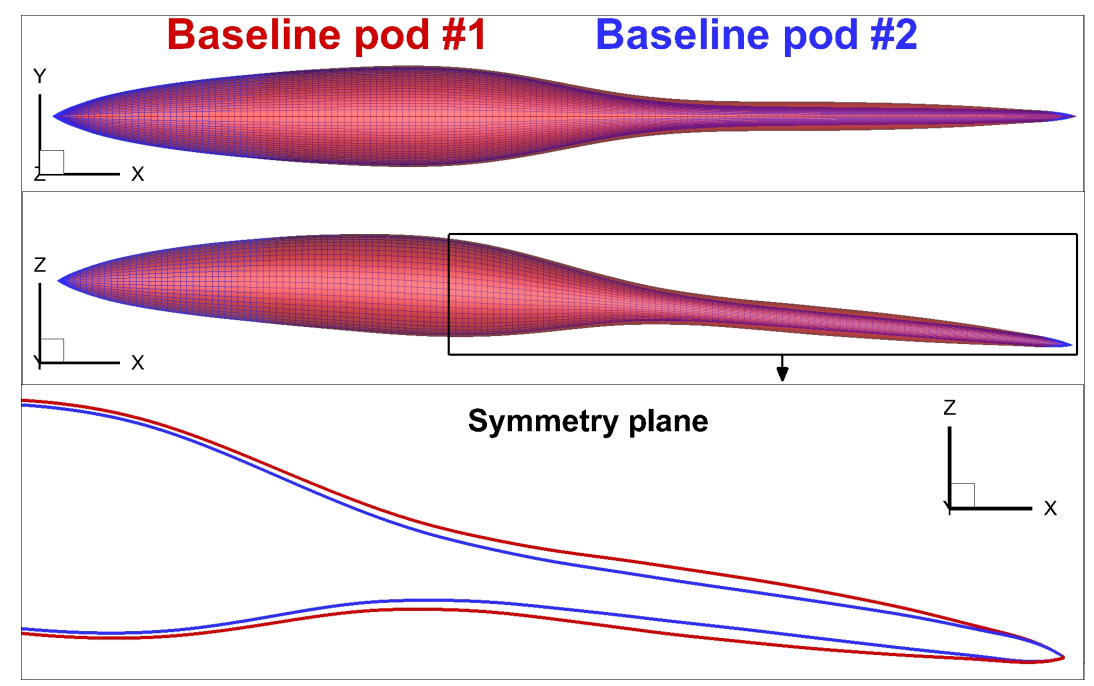

Figure A-2. Comparison of baseline tail configurations.

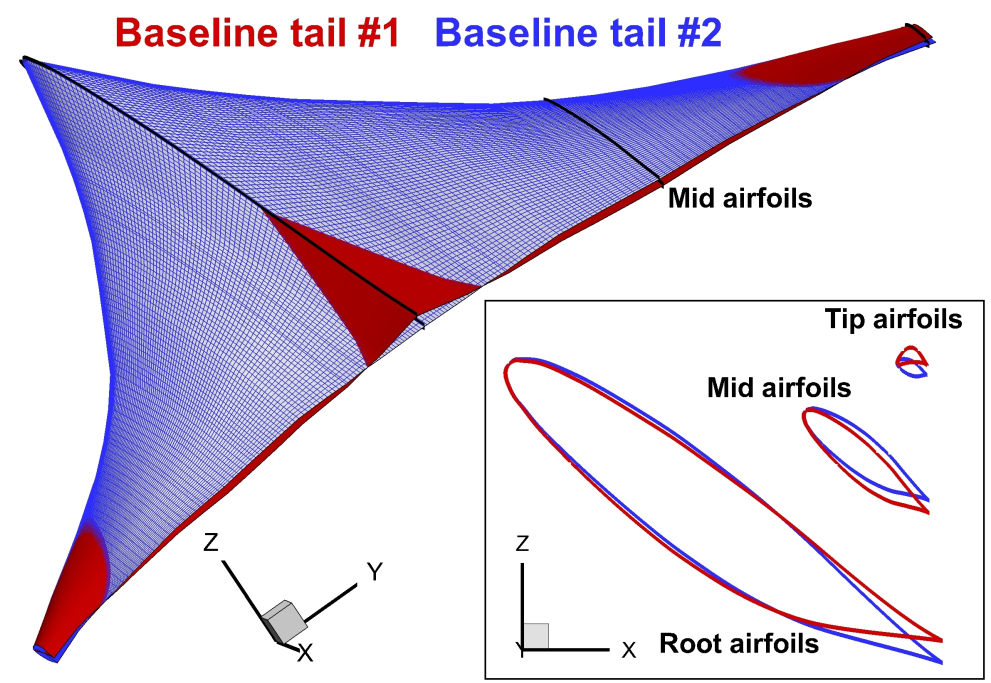

Figure A-3. Comparison of baseline tail configurations.

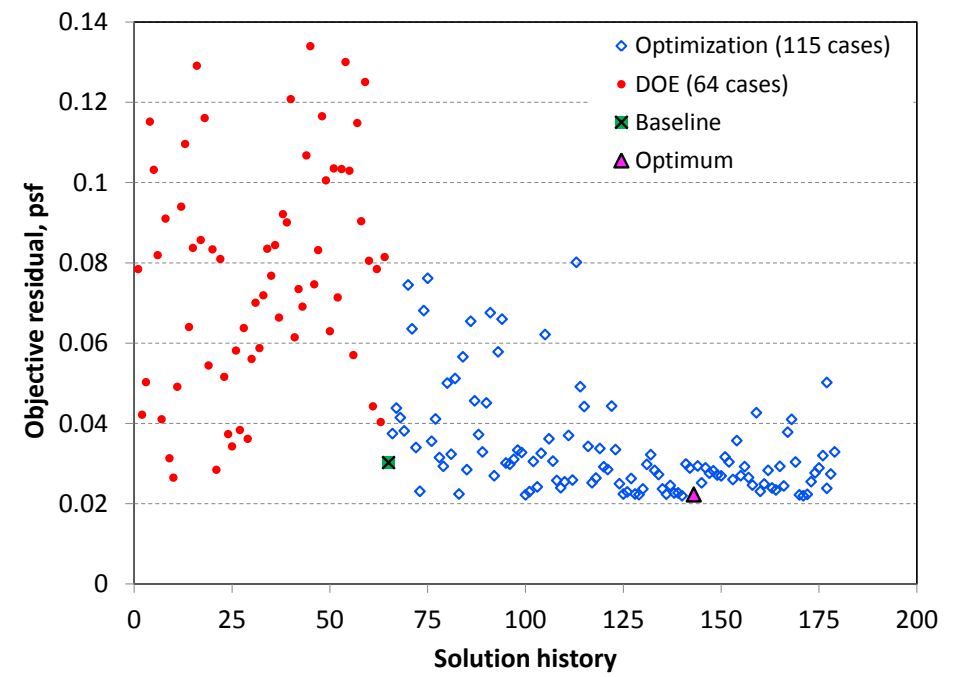

Figure A-4. Convergence history for optimization \#1. 


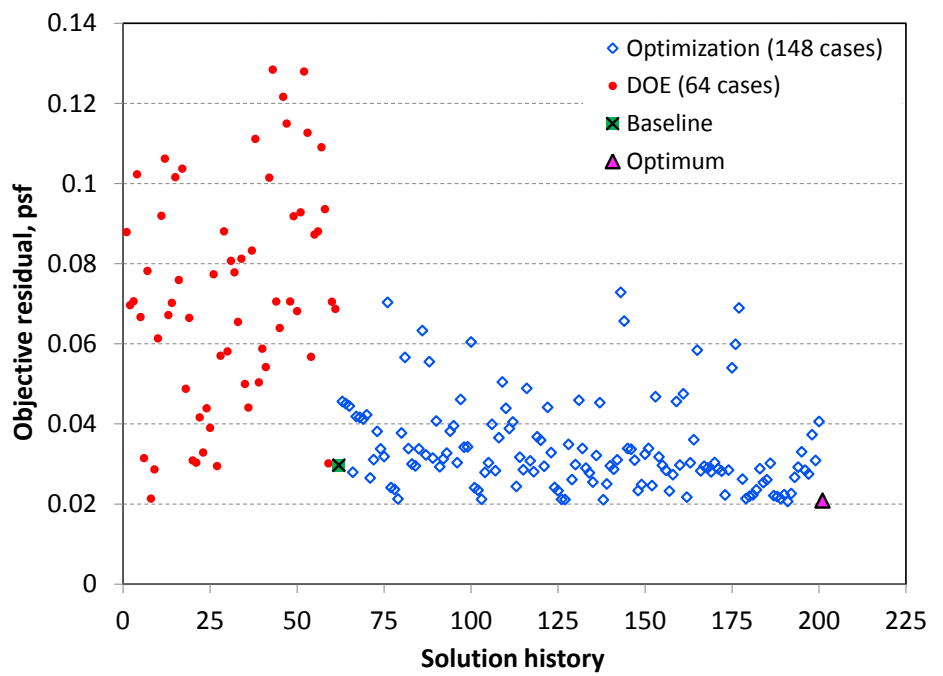

Figure A-5. Convergence history for optimization \#2.

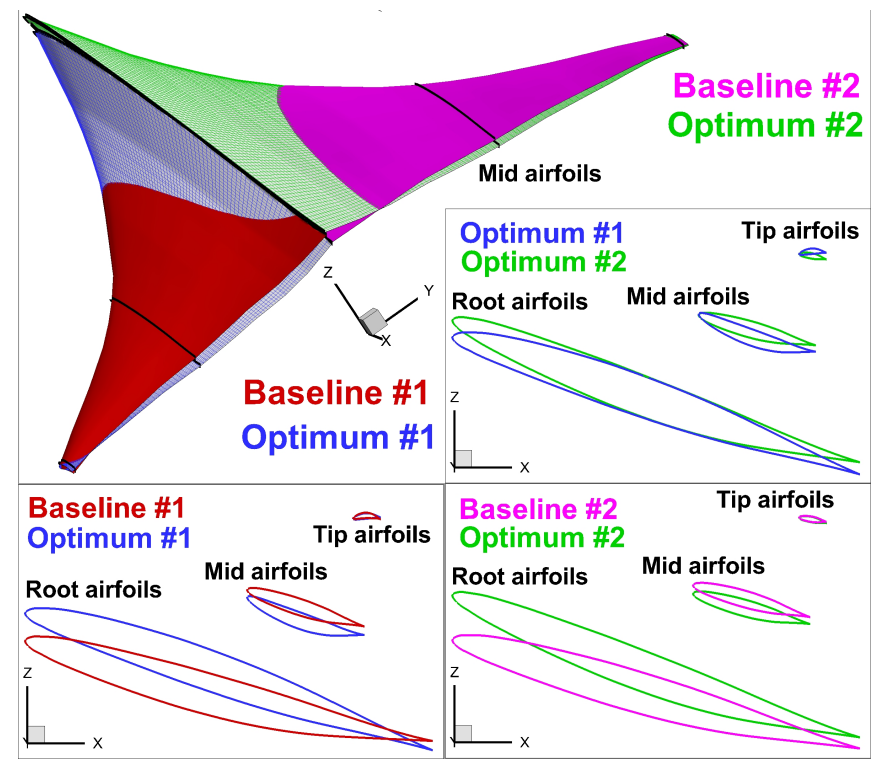

Figure A-6. Comparison of optimized horizontal tails.

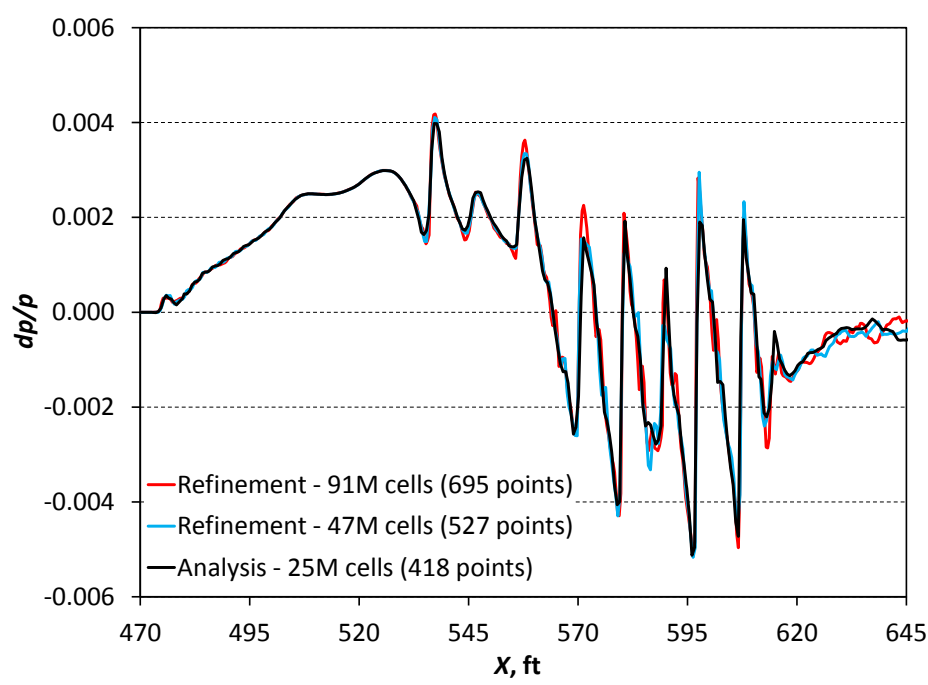

Figure A-7. Sensitivity of $d p / p$ to CFD volume mesh refinement for optimization \#2. 


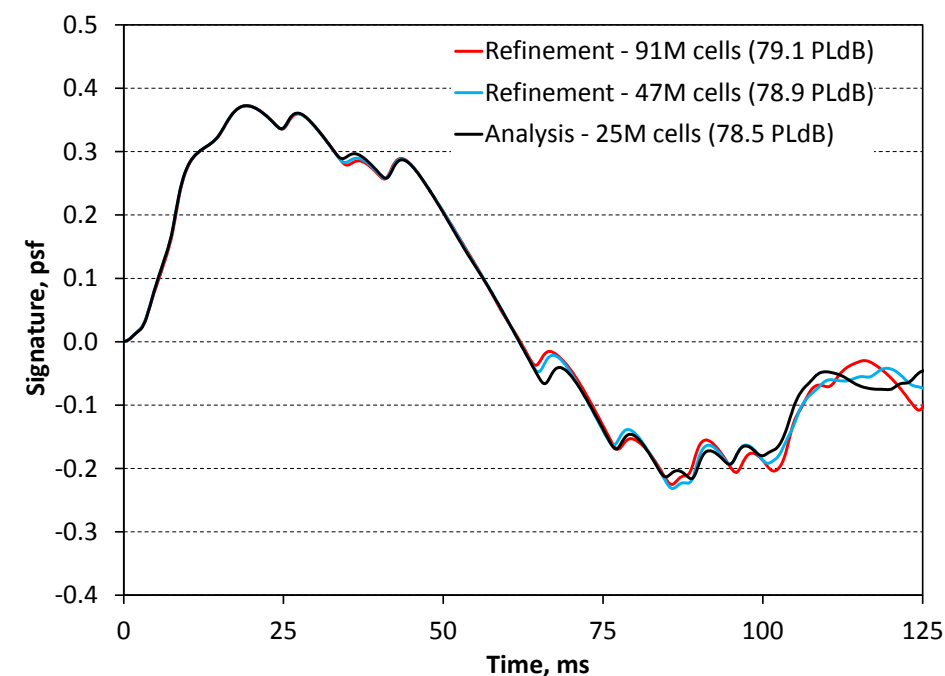

Figure A-8. Sensitivity of signature to CFD volume mesh refinement for optimization \#2. 